\title{
Enhancing Dielectric Performance of Poly(vinylidene fluoride) Nanocomposites via Controlled Distribution of Carbon Nanotubes and Barium Titanate Nanoparticles
}

\author{
Jing Wang, ${ }^{1}$ Zhicheng Shi, ${ }^{1 *}$ Xin Wang, ${ }^{1}$ Xianmin Mai, ${ }^{3 *}$ Runhua Fan, ${ }^{2}$ Hu Liu, ${ }^{4,6}$ Xiaojing Wang ${ }^{5,6}$ and Zhanhu Guo ${ }^{6 *}$
}

Polymer-based dielectric materials have attracted increasing attention owing to their huge potential applications in modern electronic devices. The dielectric behaviors of polymer composites are greatly determined by the distribution of fillers, thus the clarification of the relationship between the dielectric properties of the composites and the spatial distribution of fillers would be highly favorable for designing novel high-performance dielectrics. Herein, the dielectric performances of ternary composites consisting of barium titanate (BT), carbon nanotube (CNT) and poly(vinylidene fluoride) (PVDF) were investigated. For comparison, the dielectric properties of trilayer composites were also studied. The ternary composites exhibited an ultra-high dielectric constant of $7 \times 10^{3} @ 10 \mathrm{kHz}$, but a high loss tangent of $25 @ 10 \mathrm{kHz}$. For the trilayer composites, the BT/PVDF outer layers could restrain the development of leakage current, leading to low loss tangent $(0.03 @ 10 \mathrm{kHz})$ and high breakdown strength. Meanwhile, the trilayer composites also achieved a high dielectric constant of $95 @ 10 \mathrm{kHz}$ owing to the considerably enhanced polarizations at the filler/matrix and layer/layer interfaces. This research provides important sights into the relationship between the dielectric properties of the composites and the spatial distributions of fillers, which will strongly boost the exploration of high-performance dielectrics.

Keywords: Polymer composite; Dielectric properties; Multilayer; Percolation; High- $k$ material.

Received 21 August 2018, Accepted 10 October 2018

DOI: $10.30919 / \mathrm{es} 8 \mathrm{~d} 759$

\section{Introduction}

Functional polymer-matrix nanocomposites with high dielectric constant $\left(\varepsilon_{\mathrm{r}}\right)$ and low loss become promising candidates for various applications, such as organic field-effect transistors (OFETs), ${ }^{1,2}$ electrical stress control applications, ${ }^{3}$ energy-storage devices, ${ }^{4-7}$ antennas, ${ }^{8}$ actuators, ${ }^{9}, e^{10}$ etc., owing to their excellent dielectric and mechanical properties (e.g., high breakdown strength, low loss, flexibility, easy processability, etc.). Nonetheless, there are still challenges preventing dielectric polymers from being widely used, among which is the limited value of dielectric constant (i. e. mostly $\left.\varepsilon_{\mathrm{r}}<10\right) .{ }^{11-13}$ Currently, inorganic fillers with high permittivity (e. g.

${ }^{1}$ School of Materials Science and Engineering, Ocean University of China, Qingdao 266100, Chin.

${ }^{2}$ Institute of Marine Materials Science and Engineering, Shanghai Maritime University, Shanghai 201306, China

${ }^{3}$ School of Urban Planning and Architecture, Southwest Minzu University, Chengdu 610041, China

${ }^{4}$ National Engineering Research Center for Advanced Polymer Processing Technology,Zhengzhou University, Zhengzhou 450002, China

${ }^{5}$ School of Material Science and Engineering, Jiangsu University of Science and Technology, Zhenjiang, Jiangsu, 212003, China

${ }^{6}$ Integrated Composites Laboratory (ICL), Department of Chemical \& Biomolecular Engineering, University of Tennessee, Knoxville, TN 37996, USA

*E-mail: zcshi@ouc.edu.cn ; maixianmin@foxmail.com ; zguo10@utk.edu
$\mathrm{BN}, \mathrm{BaTiO}_{3}, \mathrm{TiO}_{2}$, or $\left.\mathrm{Ba}_{\mathrm{x}} \mathrm{Sr}_{(1-\mathrm{x})} \mathrm{TiO}_{3}\right)^{14-17}$ or conductor fillers (e.g. carbon nanotubes, graphene or metals $)^{18-21}$ are usually introduced into the polymer matrix to raise the value of $\varepsilon_{\mathrm{r}}$. However, enhanced $\varepsilon_{\mathrm{r}}$ is usually realized at the expense of increased dielectric loss because of the severe local electric field distortion caused by the enormous difference in dielectric constants between polymer matrix and fillers. $^{22-24}$

To simultaneously achieve high dielectric constant and low loss, significant efforts have been made to construct insulating shells on the filler surface to improve the dielectric compatibility between fillers and polymer matrix, hence relieves the local electric field distortion and suppresses the leakage current. ${ }^{25-35}$ Zhang et al. ${ }^{25}$ reported a high dielectric constant of about 2 times over polymer matrix in poly (vinylidene fluoride-hexafluoropropylene) (P(VDFHFP))-based dielectrics with a small loading of 7 vol\% $\mathrm{TiO}_{2} @ \mathrm{BaTiO}_{3}$ nanoparticles. Tong et al..$^{30}$ also successfully obtained dielectric films with balanced permittivity and loss via chemical modification of graphene with 4-azidotetrafluorobenzoic acid. Unfortunately, a significant drawback of aforementioned surfacemodification approaches is that the effective buffer layer can only be formed around the fillers so that the conductive paths in polymer matrix can't be blocked and thus the loss suppression effect is limited. Moreover, the dielectric constant boost effect was not obvious even if the filler faction exceeded $50 \mathrm{vol} \%{ }^{6}$

Except surface functionalization, another promising way to achieve balanced dielectric constant and loss is adjusting the spatial distribution of fillers in the matrix. For example, multilayer composites, whose dielectric performances could be easily tailored by adjusting the composition of each layer, number of layer, and 
stacking sequence, etc., ${ }^{36-42}$ were supposed to have huge potential for dielectrics with balanced dielectric constant and loss. Wang et al. ${ }^{22}$ reported greatly improved dielectric constants and maintained low loss in $\mathrm{BaTiO}_{3} / \mathrm{PVDF}$ nanocomposites consisting of three layers containing graded fractions of $\mathrm{BaTiO}_{3}$. Similarly, Liu et al. ${ }^{42}$ also achieved substantially enhanced dielectric constant and low loss in trilayer nanocomposites consisting of highly insulating PVDF/boron nitride as outer layers and intensely polarizable PVDF/barium strontium titanate as middle layers. It is indicated that the charge accumulations and polarizations at the interfaces between adjacent layers contribute to extra enhancement of permittivity. Meanwhile, suppressed leakage current loss and enhanced breakdown strength were achieved by blocking the transport of carriers between adjacent layers. Recently, Cai et $a l^{43}$ theoretically explored the effect of filler distribution on the dielectric response and breakdown strength of nanocomposites through finite element method and phase field method. Results indicated that the non-uniform distribution of fillers will aggravate the concentration of local electric field, thus slightly enhances the dielectric response but seriously decreases the breakdown strength of nanocomposites.

As discussed above, the dielectric responses of composites will be greatly determined by the distribution of fillers and it is of great interest to explore the relationship between dielectric performances and spatial distribution of fillers. Herein, a series of trilayer nanocomposites, in which different amounts of BT particles were added into the two outer layers and CNT was embedded into the middle layer, were prepared. For comparison, single-layer ternary CNT/BT/PVDF composites composed of mixed BT and CNT particles homogeneously dispersed in PVDF matrix were prepared. The dielectric analysis results indicated that the trilayer composites exhibited significantly suppressed loss, enhanced breakdown strength and impressive dielectric constants compared with single-layer ternary composites. This work experimentally presented how the spatial distribution of fillers influences the dielectric performances of composites, which offers important information for the design and exploration of high performance dielectric composites.

\section{Experimental section}

\subsection{Materials}

CNT (VGCF-H, Showa Denko K.K.), barium titanate $\left(\mathrm{BaTiO}_{3},<3\right.$ $\mu \mathrm{m},>99.5 \%$, Aladdin Industrial Corporation), polyvinylidene difluoride (PVDF, Kynar ${ }^{\circledR}$ 761A, Arkema), polyvinylpyrrolidone (PVP, K30 (GR), Sinopharm Chemical Reagent Co., Ltd.) and ethanol ( $\geq 99.7 \%$, Sinopharm Chemical Reagent Co., Ltd.) were used as received without further purification.

\subsection{Preparation of trilayer composites}

$\mathrm{BaTiO}_{3}$-PVDF mixtures were prepared as previously reported. ${ }^{44,45}$ Briefly, $\mathrm{BaTiO}_{3}$ and PVDF powders were mixed via ball-milling (300 r/min) for $30 \mathrm{~min}$ in absolute ethyl alcohol. The ball-milled mixture was dried in an oven at $70{ }^{\circ} \mathrm{C}$ for $6 \mathrm{~h}$ and ground in an agate mortar for $20 \mathrm{~min}$ to obtain the $\mathrm{BaTiO}_{3}-\mathrm{PVDF}$ mixture. For the preparation of CNT-PVDF mixture, CNT and PVP powders were mixed into ethanol in a proportion of 1:2 by vigorously tip-type sonication for $2 \mathrm{~h}$, where PVP was used as dispersant for the surface modification of CNT to improve the dispersion. Then, PVDF powders were added into the above mixture, followed by magnetic stirring for $5 \mathrm{~h}$ and ball milling at $300 \mathrm{rpm}$ for $3 \mathrm{~h}$ to form homogeneous mixture. Subsequently, the mixture was dried in an oven at $50{ }^{\circ} \mathrm{C}$ for $10 \mathrm{~h}$ and ground in an agate mortar for $30 \mathrm{~min}$ to obtain the CNT-PVDF mixture.

In trilayer composites, the outer layers (top and bottom layers) and middle layer were BT/PVDF and CNT/PVDF composites, respectively (Figure 1b). Firstly, the BT/PVDF mixture with $\mathrm{X}$ wt $\%$ BT content was poured into the hot-pressing mould and pressed under a pressure of $1 \mathrm{MPa}$ at room temperature to make the surface flat. Secondly, the CNT/PVDF mixture with $0.4 \mathrm{wt} \% \mathrm{CNT}$ content was put onto the surface of bottom layer and pressed at room temperature to form the middle layer. Subsequently, the first step was repeated to form the top layer. Finally, the three layers were hot pressed at $180{ }^{\circ} \mathrm{C}$ and $10 \mathrm{MPa}$ for $5 \mathrm{~min}$. Trilayer composites were obtained after cooling down naturally to room temperature. The diameter of every sample was $20 \mathrm{~mm}$. The thickness of each layer in a trilayer composite is $0.3 \mathrm{~mm}$, and the total thickness of a trilayer composite is $0.9 \mathrm{~mm}$. In this paper, composites with $\mathrm{X} \mathrm{wt} \% \mathrm{BT}$ in outer layers and $0.4 \mathrm{wt} \% \mathrm{CNT}$ in middle layer were reported as $\mathrm{X}$ $0.4-\mathrm{X}$.

\subsection{Preparation of single-layer ternary CNT/BT/PVDF composites}

CNT and PVP powders were first mixed into ethanol under tip-type sonication for $2 \mathrm{~h}$. After that, the BT powders were added into the suspension and sonicated for $30 \mathrm{~min}$. PVDF powders were then added into the above mixture, followed by stirring for $5 \mathrm{~h}$ and ball milling at $300 \mathrm{rpm}$ for $3 \mathrm{~h}$. The as prepared mixture was dried in an oven at $50{ }^{\circ} \mathrm{C}$ for $10 \mathrm{~h}$ and ground in an agate mortar for $30 \mathrm{~min}$ to obtain the CNT-BT-PVDF mixture. Subsequently, the CNT-BTPVDF mixtures were hot pressed at $180{ }^{\circ} \mathrm{C}$ at $10 \mathrm{MPa}$ for $5 \mathrm{~min}$ and then cooled down to room temperature to obtain the single-layer CNT/BT/PVDF composites (Fig. 1a). The single-layer ternary composite with the same composition as trilayer composite $\mathrm{X}-0.4-\mathrm{X}$ was reported as $\mathrm{S}(\mathrm{X}-0.4-\mathrm{X})$. The diameter of each sample was 20 $\mathrm{mm}$, and the thicknesses of single-layer composites were $0.3 \mathrm{~mm}$.

\subsection{Characterization and Measurements}

The morphologies of composites and elemental distributions of titanium and carbon were observed by scanning electron microscopy (SEM, S-4800, Hitachi, Ltd.) equipped with EDS. The compositions of the single-layer CNT/BT/PVDF composites were analyzed by Xray diffractometer (XRD, D8 Advance, Bruker, Ltd.).

For the dielectric measurements, gold electrodes were sputtered on both sides of the samples. One side was entirely sputtered with gold electrode, while the other side was sputtered using an annular shadow mask with internal and external diameters of $6.4 \mathrm{~mm}$ and 10 $\mathrm{mm}$, respectively. The dielectric measurements were carried out under an ac voltage of $100 \mathrm{mV}$ at room temperature in the frequency ranging from $100 \mathrm{~Hz}$ to $1 \mathrm{MHz}$ using Agilent E4980A Precision LCR Analyzer with Electrode-D of 16451B dielectric test fixture. Meanwhile, open and short compensation were performed before dielectric measurements. The permittivity was calculated by Eq. (1):

$$
\varepsilon_{\mathrm{r}}=t C_{\mathrm{p}} / A \varepsilon_{0}
$$

where $t$ is the thickness of the sample, $A$ is the area of the electrode, $C_{\mathrm{p}}$ is the parallel capacitance, $\varepsilon_{0}$ is the absolute permittivity of free space $\left(8.85 \times 10^{-12} \mathrm{~F} / \mathrm{m}\right)$. The dc breakdown strength measurements were carried out using a breakdown voltage instrument (CS2674AX, Nanjing Changsheng Instrument Co. Ltd., China) at room temperature and the characteristic leakage current was set as $0.8 \mathrm{~mA}$ during the 
tests.

\section{Results and discussions}

\subsection{Morphology and Compositions}

Fig. 1a and $\mathrm{b}$ illustrates the filler distribution of single-layer ternary and trilayer $\mathrm{CNT} / \mathrm{BT} / \mathrm{PVDF}$ composites. For single-layer ternary composites, $\mathrm{CNT}$ and $\mathrm{BT}$ were homogeneously dispersed in the whole PVDF matrix. In contrast, BT and CNT particles were confined in alternately stacked layers in the trilayer composites. Figs. $1 \mathrm{c}-\mathrm{f}$ displays the cross-sectional SEM images and EDS maps of trilayer composites. Clearly, layered structures were formed and element $\mathrm{Ti}$ was confined in outer layers (Fig. 1f). Multilayer composites, particularly those prepared by simple hot pressing process, commonly suffer from delamination phenomenon, which has been generally proved to be harmful to the dielectric and mechanical performances of multilayer materials. ${ }^{38,46,47}$ For example, Lin et $a l^{48}$ fabricated a series of trilayer structured multi-walled carbon nanotube/polyimide composites via a layer by layer casting process. It was found that obvious delamination phenomenon, as well as a dramatic increase of porosity, occurred when the MWCNT content in the middle layer reached $20 \mathrm{wt} \%$. And it was believed to be brought about by the severe aggregation of MWCNT. In this work, the trilayer composites were prepared via hot pressing three layers of mixed powder rather than rigid films. This will help the powders in the boundary region of adjacent layers make sufficient contact with each other, resulting in well unified interfacial areas. In other words, although there exist sharp "composition" boundaries between adjacent layers as demonstrated by the EDS (Fig. 1f), there is no sharp physical PVDF-PVDF boundary. As shown in Figs. 1 $\mathrm{g}-\mathrm{j}$, the single-layer ternary composite $\mathrm{S}(40-0.4-4)$ exhibits a significantly different morphology with trilayer composites. As expected, no physical layer boundary was observed and the element $\mathrm{Ti}$ was homogeneously distributed in the whole matrix without obvious sedimentation and agglomeration. Furthermore, the XRD patterns (Fig. S2) demonstrat that the CNT/BT/PVDF composites with desired compositions have been successfully obtained, and no additional phase from unexpected reactions between fillers and matrix was observed.

\subsection{Dielectric and electric properties of single-layer ternary nanocomposites}

As illustrated in Fig. 2a, the dielectric permittivity increases obviously with increasing the BT content, which should be attributed to the enhanced interfacial polarizations at enlarged BT-PVDF and BT-CNT interfaces. ${ }^{16,19}$ Particularly, a significant enhancement of permittivity from $4000 @ 10 \mathrm{kHz}$ to $7000 @ 10 \mathrm{kHz}$ was observed when the BT content increased from 60 to $70 \mathrm{wt} \%$ which should be ascribed to the formation of percolative BT networks and severely enlarged interfaces within the composites. As shown in Fig. 2b, The $\sigma_{\mathrm{ac}}-f$ relationships of the composites follow the Jonscher power law as shown by Eq. (2): ${ }^{49}$

$$
\sigma_{\mathrm{ac}}=\sigma_{\mathrm{dc}}+A(2 \pi f)^{\mathrm{s}}
$$

where $\sigma_{\mathrm{dc}}$ is the dc conductivity (frequency independent plateau in the low frequency region), $f$ is the frequency, $A$ is the preexponential factor, and $s$ is the fractional exponent. The $\sigma_{\mathrm{dc}}$ plateau indicates a metal-like conduction mechanism at low frequency and the $\sigma_{\text {ac }}-f$ relationship that agrees well with the power law $\sigma_{\text {ac }} \sim(2 \pi f)^{\mathrm{s}}$ represents a hopping conduction mechanism. ${ }^{50}$ Fig. $2 \mathrm{c}$ displays the frequency dispersions of imaginary part of permittivity. Surprisingly, higher BT content, which notably boosted the dielectric permittivity (Fig. 2a), results in a distinct decrease of dielectric loss. As we know, enhanced dielectric permittivity was always accompanied by elevated loss. However, enhanced permittivity and suppressed loss were simultaneously achieved in our current CNT and BT co-filled ternary nanocomposites. As mentioned above, the enhanced

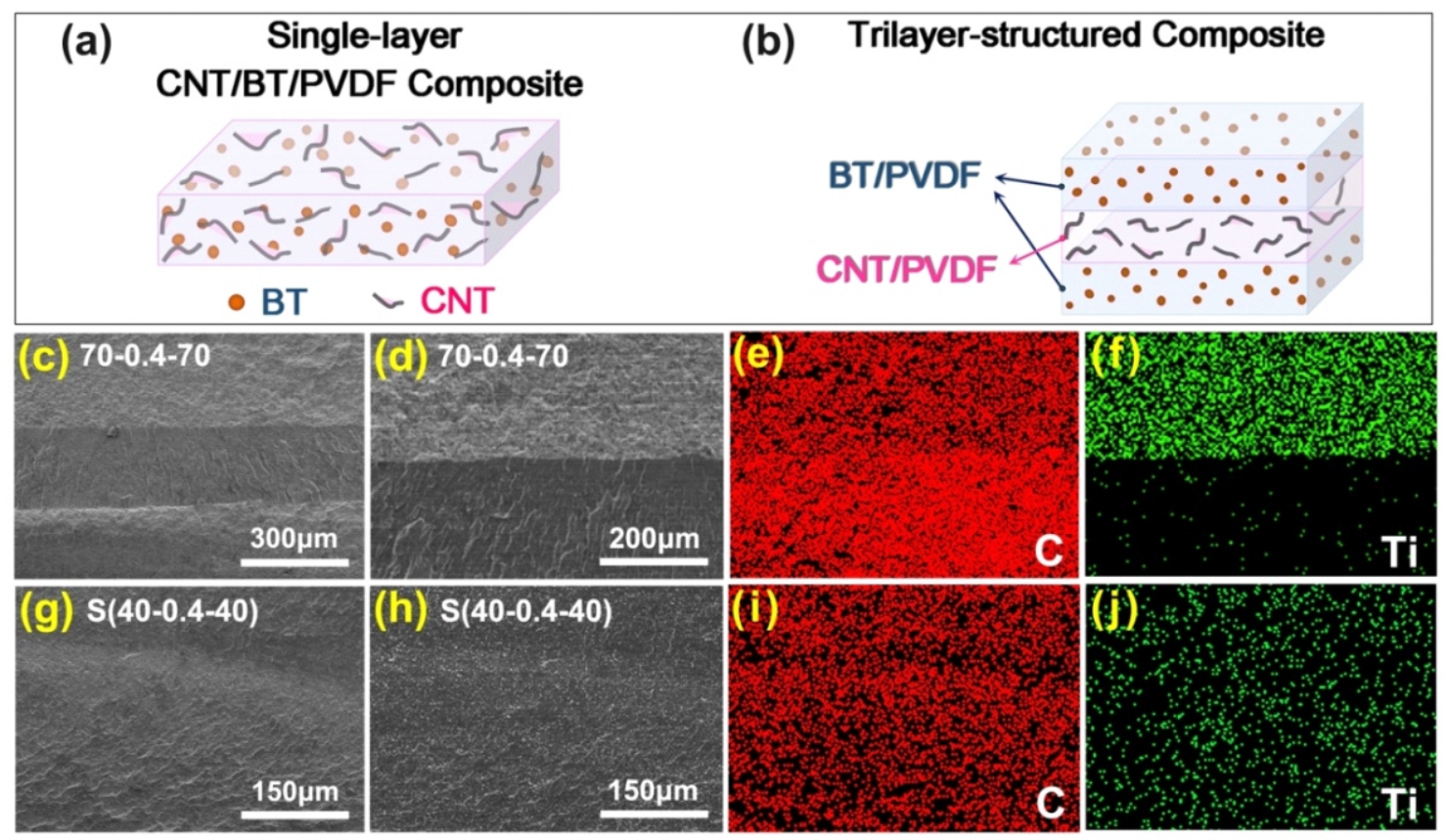

Fig. 1 Schematic illustrations of (a) single-layer CNT/BT/PVDF composites and (b) trilayer composites. (c, d) Cross-sectional SEM images and (e, f) EDS maps of 70-0.4-70 composites. (g, h) Cross-sectional SEM images and (e, f) EDS maps of single-layer S(40-0.4-40) composites. 
permittivity is brought about by interfacial polarizations at the BTPVDF and BT-CNT interfaces. Then the mechanism behind the suppressed loss with increasing the BT content will be discussed. As we know, the dielectric loss describes the energy dissipation in a dielectric material because of conduction, dipole polarizations, and interfacial polarizations, which can be described by the following Eq. (3): $:^{3,10}$

$$
\varepsilon^{\prime \prime}=\varepsilon_{\mathrm{dc}}^{\prime \prime}+\varepsilon_{\mathrm{MW}}^{\prime \prime}+\varepsilon_{\mathrm{D}}^{\prime \prime}
$$

where $\varepsilon_{\mathrm{dc}}^{\prime \prime}, \varepsilon^{\prime \prime}{ }_{\mathrm{MW}}$ and $\varepsilon_{\mathrm{D}}^{\prime \prime}$ are conduction loss, interfacial polarization loss and dipole loss, respectively. The conduction loss $\varepsilon^{\prime \prime}{ }_{\mathrm{dc}}$ can be expressed as:

$$
\varepsilon_{\mathrm{dc}}^{\prime \prime}=\frac{\sigma_{\mathrm{dc}}}{2 \pi f}
$$

where $\sigma_{\mathrm{dc}}$ and $f$ represent the direct current conductivity and frequency, respectively. As shown in Fig. $2 \mathrm{c}$, the $\varepsilon "-f$ relationships agree well with Eq. (4), indicating that the loss of the ternary nanocomposites was dominated by conduction loss. It is generally believed that the high-frequency loss is mainly associated with dipolar relaxation, whereas the low frequency loss is dominated by interfacial polarization and leakage conduction. ${ }^{29}$ Accordingly, the slight deviation of experimental results from the linear $\sigma_{\mathrm{dc}} \sim 1 / f$ relationship at high frequency region indicates the increased contribution of polarization loss to the overall loss. As illustrated in Fig. 2e, before filling the BT particles, CNT networks will be formed once the CNT content exceeds the percolation threshold, leading to significantly elevated permittivity, conductivity and loss (Fig. S4). After filling the BT particles, a large fraction of BT nanoparticles will be located between CNT fillers and thus inhibit the CNT bridge formation due to the random dispersion of BT particles. Therefore, the formation of CNT networks was hindered and the conduction loss was suppressed. However, the inhibition effect is limited because the dispersion of CNT and BT is not absolutely homogenous. For instance, if the aggregation of CNT fillers is severe, the addition of BT fillers may not isolate the CNT but lower the percolation threshold of CNT/PVDF system and facilitate the formation of CNT networks, hence increase the conduction loss. ${ }^{51}$ Hopefully, the loss of the ternary composites could be further
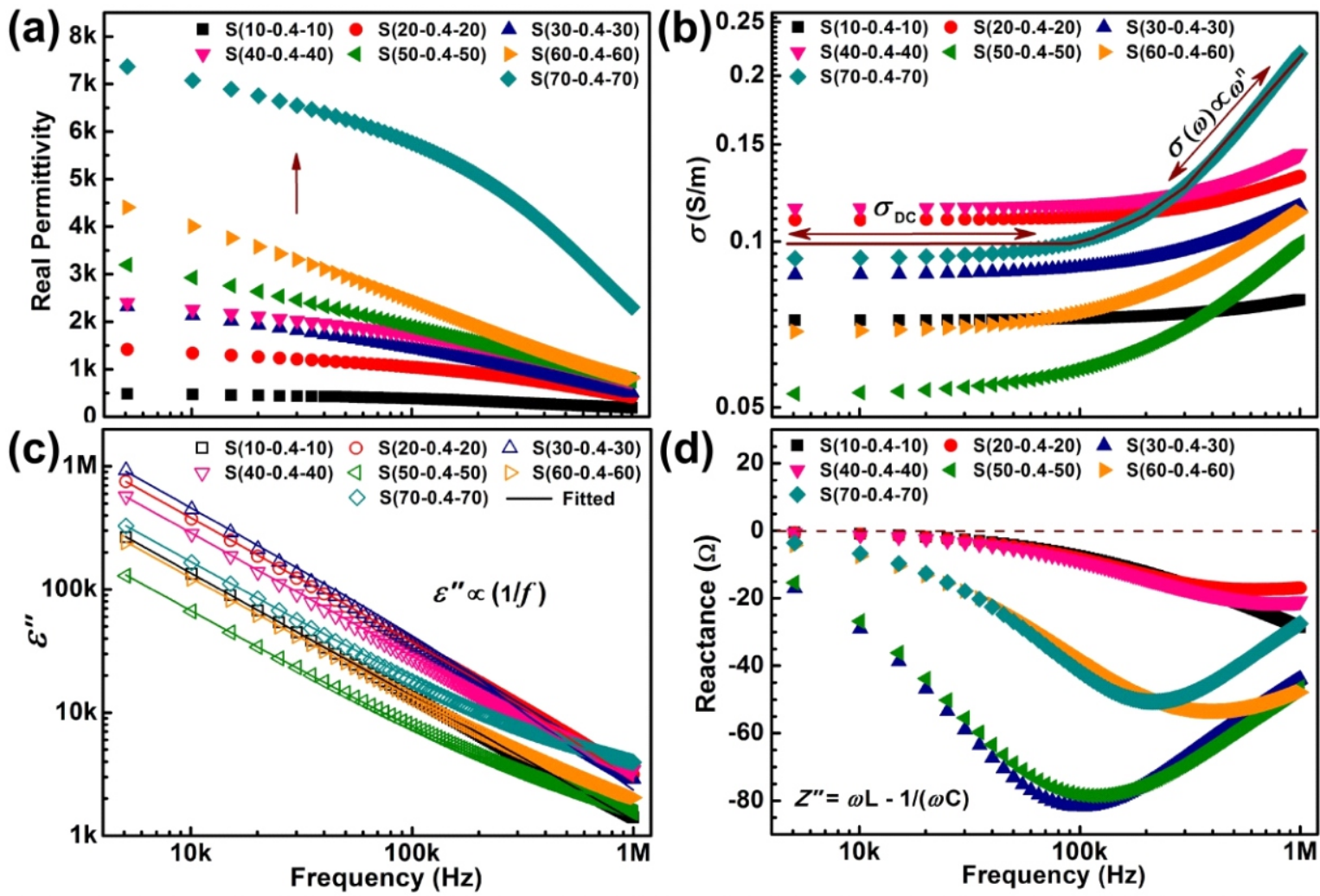

(e)
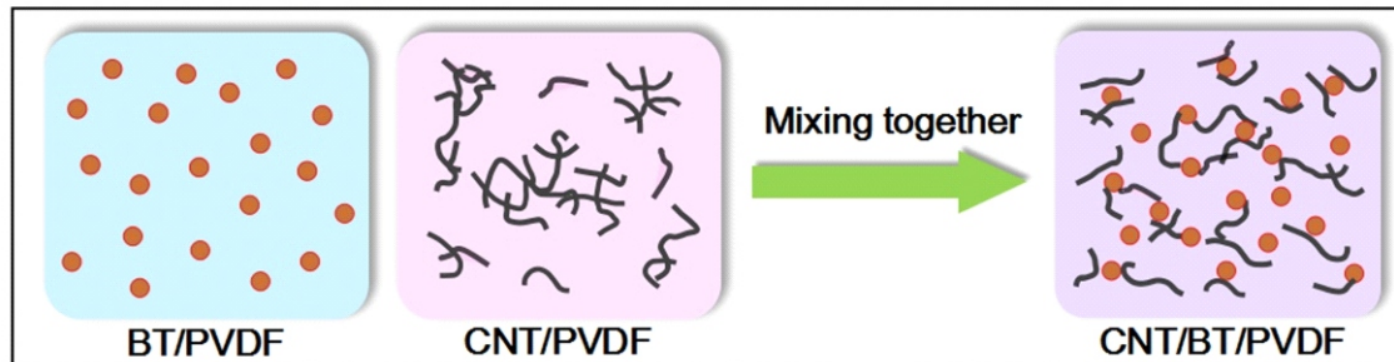

Fig. 2 Frequency dependences of (a) permittivity, (b), conductivity, (c) imaginary part of permittivity and (d) reactance for single-layer CNT/BT/PVDF composites with the same comparisons corresponding to the trilayer composites; (e) Schematic microstructure of BT/PVDF, $\mathrm{CNT} / \mathrm{PVDF}$ and ternary CNT/BT/PVDF composites. 
suppressed via guaranteeing the uniform distribution of fillers, thus make them promising candidates for embedded capacitors. Composites under electric fields are equivalent to electrical systems, in which reactance $Z^{\prime \prime}$ (the opposition of a circuit element to a change of current or voltage due to that element's inductance or capacitance) may provide some important clues regarding the electrical response of composites. Generally, the reactance can be expressed as $Z^{\prime \prime}=\omega L-1 /(\omega C)$, where $\omega L$ and $-1 /(\omega C)$ represent the inductive reactance and capacitive reactance, respectively. As shown in Fig. 2d, all the ternary nanocomposites manifest negative reactance, characterizing the capacitive behavior. For typical capacitive materials, such as BT/PVDF composites, the $Z^{\prime \prime}-f$ curves follow $Z^{\prime \prime}=-1 /(\omega C)$ (Fig. S5). However, the $Z^{\prime \prime}$ of the ternary nanocomposites decreases at low frequency and then increases at high frequency with increasing the frequency (Fig. 2d), indicating that both capacitive reactance and inductive reactance exist in the composites. Additionally, the $Z^{\prime \prime}$ values of ternary nanocomposites are almost 5 orders of magnitude lower than that of BT/PVDF composites. Such very small capacitive reactance should be attributed to the large capacitance $C$ generated by ultrahigh dielectric permittivities (Fig. 2a) as well as the inductive reactance brought about by conductive networks in composites.

\subsection{Dielectric and electric properties of trilayer nanocomposites}

Besides designing ternary composites, the spatial distribution of fillers could also be adjusted via constructing multilayer structures. As shown in Fig. 3, the dielectric permittivity of trilayer composites increases with increasing the BT fraction, which should be attributed to the Maxwell-Wagner-Sillars polarizations ${ }^{52}$ at the enlarged BT/PVDF interfaces. Moreover, the dielectric permittivity decreases
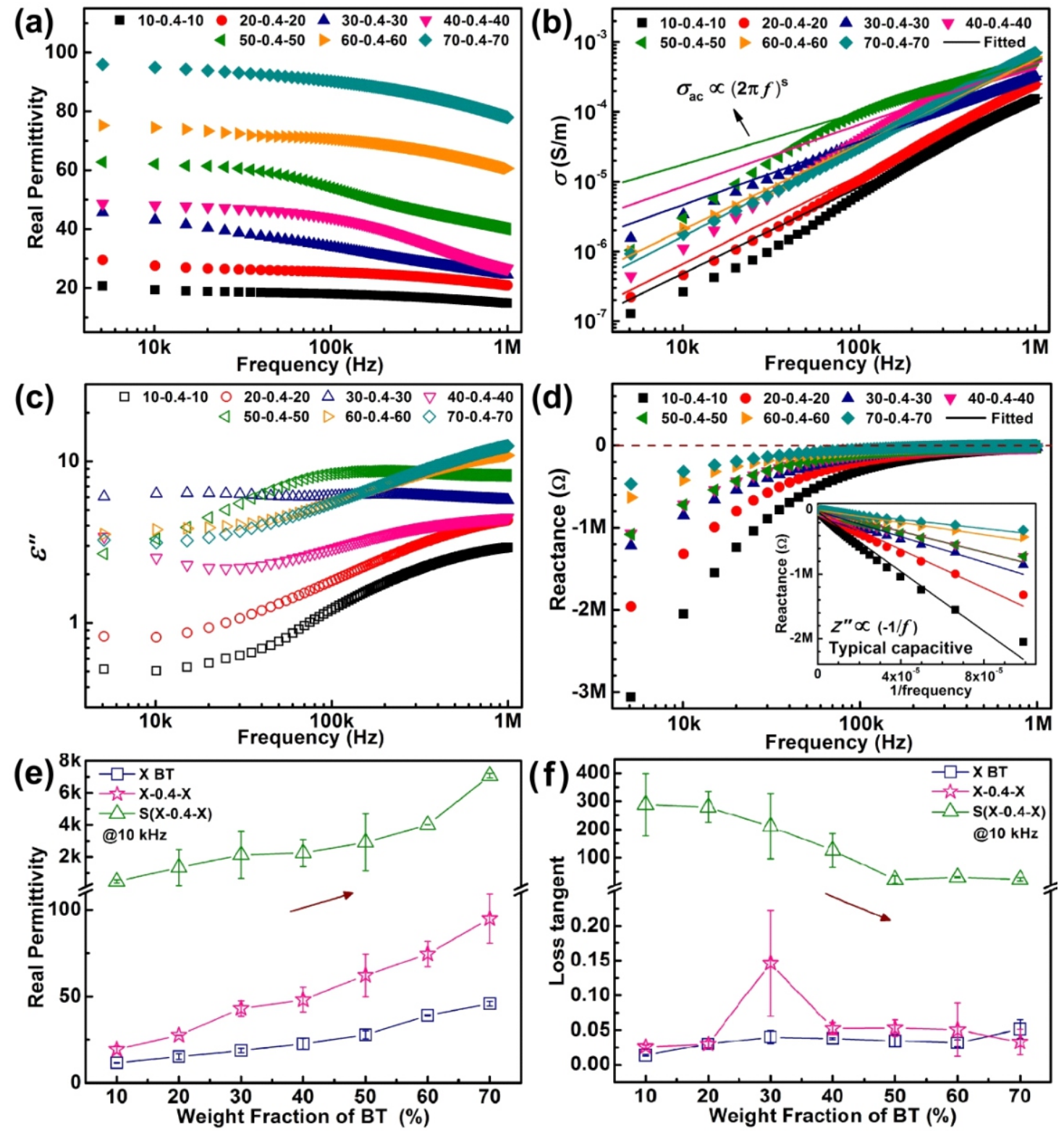

Fig. 3 Frequency dependences of (a) permittivity, (b), conductivity, (c) imaginary part of permittivity and (d) reactance for trilayer composites with different BT contents; Variation of (e) permittivity and (f) loss tangent with a BT weight fraction for BT/PI composites, CNT/BT/PVDF ternary composites and trilayer composites. 
with increasing the frequency. Generally, when a composite was put under an external AC electric field, various types of polarizations (e.g., electronic, vibrational, dipolar and space charge polarizations) occur at low frequencies, ${ }^{3,10}$ the polarizations could keep up with the change speed of external AC electric field, and the composites manifest high permittivity. However, some types of polarizations will lag behind the $\mathrm{AC}$ electric field once the frequency of external electric field exceeds some critical value, leading to the decrease of permittivity with increasing the frequency (Fig. 3a). In this work, an obviously boosted dielectric permittivity of $95 @ 10 \mathrm{kHz}$, which is about twice of the dielectric permittivity of the BT/PVDF composite containing 70 wt\% BT (about 46@10 kHz, Figure S5), was obtained in the 70-0.4-70 trilayer composites. Meanwhile, a very low loss tangent of 0.032 was achieved in the 70-0.4-70 trilayer composites, which is comparable to the loss of BT/PVDF composites and much lower than the loss of CNT/PVDF composites (Fig. S5). As presented in Fig. 3b, the conductivity $\sigma_{\text {ac }}$ increases with increasing the frequency and the frequency dispersion behaviors agree well with the power law $\sigma_{\mathrm{ac}} \sim(2 \pi f)^{\mathrm{s}}$, indicating that the nanocomposites follow the hopping conduction rather than the insulator-conductor transition. Figure $3 \mathrm{c}$ displays the frequency dependences of imaginary part of permittivity for trilayer composites. Different from the ternary nanocomposites, the loss of trilayer composites is mainly originated from the interfacial polarization and dipole polarization. As shown in Fig. 3c, the loss gradually increases with increasing the frequency due to the fact that some types of polarizations with long relaxation time cannot keep up with the fast change of electric field direction. ${ }^{16,19}$ Fig. $3 \mathrm{~d}$ presents the frequency dispersions of reactance $Z^{\prime \prime}$. Unlike the ternary nanocomposites, the frequency dispersions of reactance for that of the BT/PVDF composites, indicating a typical capacitive characteristic. In other words, although the ternary nanocomposites exhibit ultrahigh dielectric permittivity, the trilayer composites with superior overall dielectric performances would be better candidates for capacitors. To obtain an intuitive comparison of dielectric performances of single-layer and trilayer composites, the dielectric constants and loss tangents (@10 kHz) of BT/PVDF, $\mathrm{CNT} / \mathrm{BT} / \mathrm{PVDF}$ trilayer composites agree well with $Z^{\prime \prime}=-1 / \omega \mathrm{C}$ and the magnitude of reactance is comparable to and trilayer composites as a function of BT content were presented in Fig. 3. As can be seen, the dielectric constants of both single-layer and trilayer composites increase evidently with increasing the BT content. The single-layer CNT/BT/PVDF composites exhibit much higher dielectric constants than the single-layer BT/PVDF and trilayer CNT/BT/PVDF composites, whereas the partial formation of CNT networks gives rise to undesired increase of loss. For the trilayer composites, there are additional barrier interfaces between adjacent layers which will hinder the transport of electrons, thus suppresses the leakage current and loss. Comparing the three groups of composites, the trilayer composites exhibit superior overall dielectric performance with moderate dielectric permittivity and low loss, which could be promising candidates for capacitors, antennas and field-effect transistors, etc. $^{2,7,8}$

\subsection{Breakdown strength of trilayer composites}

Breakdown strength (BDS) is another crucial parameter for dielectric materials. Generally, the dilemma of dielectric composites is that the improvement of dielectric constant is always achieved at the cost of sharply reduced breakdown strength. Consequently, the reasonable balance between dielectric constant and breakdown strength is the key to promote their potential applications. Herein, the failure probabilities of the trilayer composites were measured and analyzed using the two-parameter Weibull distribution, which is described by Eq. (5): ${ }^{53}$

$$
P(E)=1-\exp \left[-\left(\frac{E}{\alpha}\right)^{\beta}\right]
$$

where $P(E)$ is the cumulative failure probability, $E$ is the experimentally tested breakdown electric field, $\alpha$ is the electric field for which there is a $63.2 \%$ probability of sample breakdown (Weibull breakdown strength, BDS), and $\beta$ is a shape parameter or the slope of the derived logarithm function representing the scatter of the tested BDS. When the $\beta$ value is 3 , the tested data follow Gaussian distribution, and a higher value of $\beta$ indicates a higher level of reliability. Fig. 4a presents the Weibull distribution plot of the breakdown strength of the trilayer composites with varied BT loadings measured at room temperature. With increased the BT loading, the BDS of trilayer composites increases gradually and then decreases, yielding the highest BDS of $242.5 \mathrm{kV} / \mathrm{cm}$ in the $60-0.4-60$ composites. In a composite, increasing filler loading will bring about two contradictory consequences. One is the worsened dispersion of
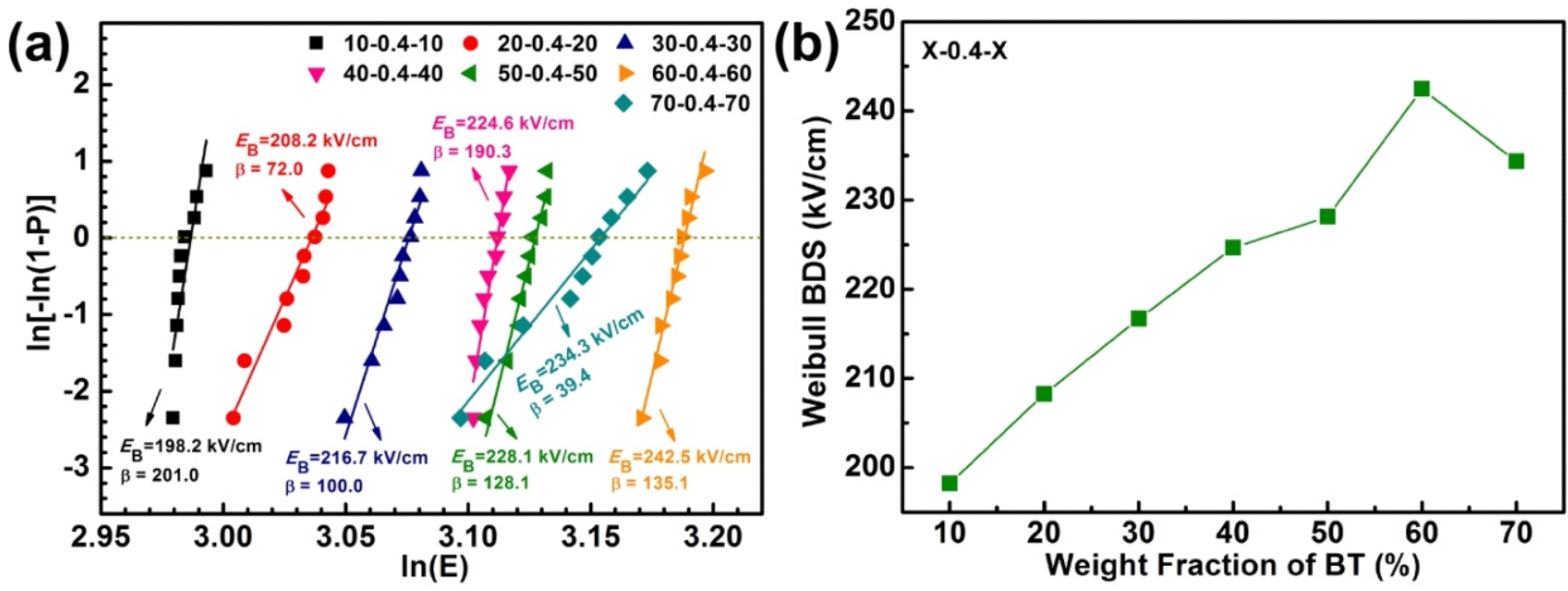

Fig. 4 Weibull distribution of breakdown strengths (BDS) for trilayer composites with different BT contents in outer layers (a); variation of Weibull BDS of trilayer composites as a function of BT loading (b). 
fillers, which is detrimental to BDS. The other is increased length of electric tree development paths, which is beneficial to high BDS. ${ }^{14,22,41,43}$ The competition between such two factors varies with increasing the filler content, yielding the non-monotonic relationship between BDS and filler content. Similar variation trend of BDS with increasing the BT content was also reported in other BT filled polymer composites, which was ascribed to the more serious structural imperfection phenomena caused by the porosity, micron voids and cracks when a critical fraction of fillers are employed into the polymer matrix. ${ }^{41,42}$ In multilayer composites, more electric voltage will concentrate on the layer with lower permittivity and thus protect the layer with high permittivity from early breakdown. ${ }^{40}$ In our current trilayer composites, although the CNT/PVDF layer is easy to be broken down, the majority of the applied electric voltage will concentrate on the outer BT/PVDF layers which possess higher BDS, thus relieves the electric field strength in the middle layer. On the other hand, there exists a gap of electric field strength at the interfaces between BT/PVDF and CNT/PVDF layers. Consequently, the development of electric trees has to suffer from sharp decrease (or increase) of electric field which is beneficial for blocking the breakdown. In other words, the interfaces between adjacent layers can effectively prevent the electric trees from penetrating the entire trilayer composites and thus ensure the satisfactory breakdown strength. $^{22,40}$

\section{Conclusions}

With the aim of investigating the impacts of filler distributions on dielectric performances, single-layer and trilayer composites were designed and prepared. The redistribution of electric field occurred when the spatial distributions of fillers changed, leading to the variation of dielectric constant, loss and breakdown strength. Compared with single-layer CNT/PVDF and BT/PVDF composites, the CNT and BT co-filled single-layer ternary composites as well as the trilayer composites were demonstrated to be capable of balancing enhanced dielectric constant and low loss simultaneously. In addition, the trilayer composites possess breakdown strengths superior to CNT and BT co-filled ternary composites. In the trilayer composites, the interfaces between adjacent layers can not only induce intense interfacial polarizations but also act as effective barriers to the formation and growth of conductive paths throughout the composites, thus yielding significantly enhanced dielectric constant without sacrificing the low loss and high breakdown strength. This study revealed the critical role of the filler distributions in the dielectric properties, which is of great significance to promote the exploration of nanocomposites with excellent dielectric performances.

\section{Acknowledgements}

This work was supported by National Natural Science Foundation of China (51773187, 51402271), Foundation for Outstanding Young Scientist in Shandong Province (BS2014CL003).

\section{References}

1. S. K. Hwang, I. Bae, S. M. Cho, R. H. Kim, H. J. Jung and C. Park, Adv. Funct. Mater., 2013, 23, 5484-5493.

2. M. W. Si, C. S. Jiang, W. Chung, Y. C. Du, M. A. Alam and P. D. Ye, Nano Lett., 2018, 18, 3682-3687.

3. Z. M. Dang, J. W. Yuan, J. W. Zha, T. Zhou, S. T. Li and G. H. Hu, Prog. Mater. Sci., 2012, 57, 660-723.
4. F. He, S. Lau, H. L. Chan and J. T. Fan, Adv. Mater., 2009, 21, $710-715$.

5. J. K. Yuan, A. Luna, W. Neri, C. Zakri, T. Schilling, A. Colin and P. Poulin, Nat. Commun., 2015, 6, 8700-8707.

6. S. B. Luo, Y. B. Shen, S. H. Yu, Y. J. Wan, W. H. Liao, R. Sun and C. P. Wong, Energy Environ. Sci., 2017, 10, 137-144.

7. C. Zhang, Z. C. Shi, F. Mao, C. Q. Yang, J. Yang, X. T. Zhu and H. Zuo. J. Mater. Sci., 2018, 53, 13230-13242.

8. T. Inui, H. Koga, M. Nogi, N. Komoda and K. Suganuma, $A d v$. Mater., 2015, 27, 1112-1116.

9. R. M. Mutiso and K. I. Winey, Prog. Polym. Sci., 2015, 40, 63-84.

10. X. Y. Huang and P. K. Jiang. Adv. Mater., 2015, 27, 546-554.

11. C. Yang, S. J. Hao, S. L. Dai and X. Y. Zhang, Carbon, 2017, 117, 301-312.

12. F. Liang, L. Zhang, W. Z. Lu, Q. X. Wan and G. F. Fan, Appl. Phys. Lett., 2016, 108, 072902-072905.

13. L. Zhang, Z. Liu, X. Lu, G. Yang, X. Y. Zhang and Z. Y. Cheng, Nano Energy, 2016, 26, 550-557.

14. M. Donnay, S. Tzavalas and E. Logakis, Compos. Sci. Technol., 2015, 110, 152-158.

15. H. Tang, Y. Lin and H. A. Sodano, Adv. Energy Mater., 2013, 3, 451-456.

16. X. Zhang, W. W. Chen, J. J. Wang, Y. Shen, L. Gu, Y. H. Lin and C. W. Nan, Nanoscale, 2014, 6, 6701-6709.

17. H. Tang and H. A. Sodano, Nano Lett., 2013, 13, 1373-1379.

18. A. Ameli, S. Wang, Y. Kazemi, C. B. Park and P. Pötschke, Nano Energy, 2015, 15, 54-65.

19. H. Luo, K. C. Zhou, C. Bowen, F. Q. Zhang, A. Q. Wei, Z. Wu, C. Chen and D. Zhang, Adv. Mater. Interfaces, 2016, 3, 1600157-1600162.

20. S. H. Cho, J. S. Lee and J. Jang, ACS Appl. Mater. Interfaces, 2015, 7, 9668-9681.

21. L. A. Fredin, Z. Li, M. T. Lanagan, M. A. Ratner and T. J. Marks, Adv. Funct. Mater., 2013, 23 3560-3569.

22. Y. F. Wang, J. Cui, Q. B. Yuan, Y. J. Niu, Y. Y. Bai and H. Wang, Adv. Mater., 2015, 27, 6658-6663.

23. X. Zhang, Y. Shen, Q. H. Zhang, L. Gu, Y. H. Hu, J. W. Du, Y. H. Lin and C. W. Nan, Adv. Mater., 2015, 27, 819-824.

24. F. Mao, Z. C. Shi, J. Wang, C. Zhang, C. Q. Yang and M. H. Huang, Adv. Compos. Hybrid. Mater., 2018, 1, 548-557.

25. X. Zhang, Y. Shen, B. Xu, Q. H. Zhang, L. Gu, J. Y. Jiang, J. Ma, Y. H. Lin and C. W. Nan, Adv. Mater. 2016, 28, 2055-2061.

26. H. Tang, P. Wang, P. L. Zhen and X. B. Liu, Compos. Sci. Technol. 2016, 123, 134-142.

27. K. Yang, X. Y. Huang, M. Zhu, L. Y. Xie, T. Tanaka and P. K. Jiang, ACS Appl. Mater. Interfaces, 2014, 6, 1812-1822.

28. S. H. Liu and J. W. Zhai, J. Mater. Chem. A, 2015, 3, 1511-1517.

29. G. Q. Zhang, D. Brannum, D. X. Dong, L. X. Tang, E. Allahyarov, S. Tang, K. Kodweis, J. Lee and L. Zhu, Chem. Mater., 2016, 28, 4646-4660.

30. W. S. Tong, Y. H. Zhang, Q. Zhang, X. L. Luan, F. Z. Lv, L. P. Liu and Q. An, Adv. Funct. Mater., 2015, 25, 7029-7037.

31. Z. Chen, L. Y. Xie, X. Y. Huang, S. T. Li and P. K. Jiang, Carbon, 2015, 95, 895-903.

32. K. Yang, X. Y. Huang, J. L. He and P. K. Jiang, Adv. Mater. Interfaces, 2015, 2, 1500361-1500370.

33. Q. K. Guo, Q. Z. Xue, T. T. Wu, X. L. Pan, J. Q. Zhang, X. F. Li and L. Zhu, Composites: Part A, 2016, 87, 46-53.

34. P. Xu, H. G. Gui, X. X. Wang, Y. D. Hu and Y. S. Ding, Compos. 
Sci. Technol., 2015, 117, 282-288.

35. Y. J. Wan, P. L. Zhu, S. H. Yu, W. H. Yang, R. Sun, C. P. Wong and W. H. Liao, Compos. Sci. Technol., 2017, 141, 8-55.

36. P. H. Hu, Y. Shen, Y. H. Guan, X. S. Zhang, Y. S. Lin, Q. M. Zhang, C. W. Nan. Adv. Funct. Mater., 2014, 24, 3172-3178.

37. B. H. Wang, L. M. Liu, L. Z. Huang, L. F. Chi, G. Z. Liang, L. Yuan and A. J. Gu, Carbon, 2015, 85, 28-37.

38. M. H. Duduta, R. J .Wood and D. R. Clarke, Adv. Mater. 2016, 28, 8058-8063.

39. X. N. Zhu, T. T. Gao, X. Xu, W. Z. Liang, Y. Lin, C. L. Chen and X. M. Chen, ACS Appl. Mater. Interfaces, 2016, 8, 22309-22315.

40vC. Q. Yang, Z. C. Shi, C. Zhang and R. H. Fan, Mater. Lett., 2018, 231, 87-90.

41. J. Wang, Z. C. Shi, F. Mao, X. Wang, K. Zhang and J. Shi, RSC Adv., 2016, 6, 43429-43435.

42. F. H. Liu, Q. Li, J. Cui, Z. Y. Li, G. Yang, Y. Liu, L. J. Dong, C. X. Xiong, H. Wang and Q. Wang, Adv. Funct. Mater., 2017, 27, 1606292.

43. Z. M. Cai, X. H. Wang, B. C. Luo, W. Hong, L. W. Wu and L. T. Li, Compos. Sci. Technol., 2017, 145, 105-113.
44. J. Wang, Z. C. Shi, F. Mao, S. G. Chen and X. Wang, ACS Appl. Mater. Interfaces, 2017, 9, 1793-1800.

45. Z. C. Shi, J. Wang, F. Mao, C. Q. Yang, C. Zhang and R. H. Fan, J. Mater. Chem. A, 2017, 5, 14575-14582.

46. K. Z. Yin, Z. Zhou, D. E. Schuele, M. Wolak, L. Zhu and E. Baer, ACS Appl. Mater. Interfaces, 2016, 8, 13555-13566.

47. Y. Shen, Y. H. Hu, W. W. Chen, J. J. Wang, Y. H. Guan, J. W. Du, X. Zhang, J. Ma, M. Li, Y. H. Lin, L. Q. Chen and C. W Nan, 2015, Nano Energy, 18, 176-186.

48. Y. Q. Chen, B. P. Lin, X. Q. Zhang, J. C. Wang, C. W. Lai, Y. Sun, Y. R. Liu and H. Yang, J. Mater. Chem. A, 2014, 2, 14118-14126.

49. A. K. Jonscher, J. Phys. D: Appl. Phys., 1999, 32, 57-70.

50. J. C. Dyre and T. B. Schrøder, Rev. Mod. Phys., 2000, 72, 873-892.

51. H. Y. Wang, Q. Fu, J. Q. Luo, D. M. Zhao, L. H. Luo and W. P. Li, Appl. Phys. Lett., 2017, 110, 242902.

52. R. Tamura, E. Lim, T. Manaka and M. Iwamoto, J. Appl. Phys., 2006, 100, 114515.

53. E. Tuncer, D. R. James, I. Sauers, A. R. Ellis and M. O. Pace, J. Phys. D: Appl. Phys., 2006, 39, 4257-4268. 